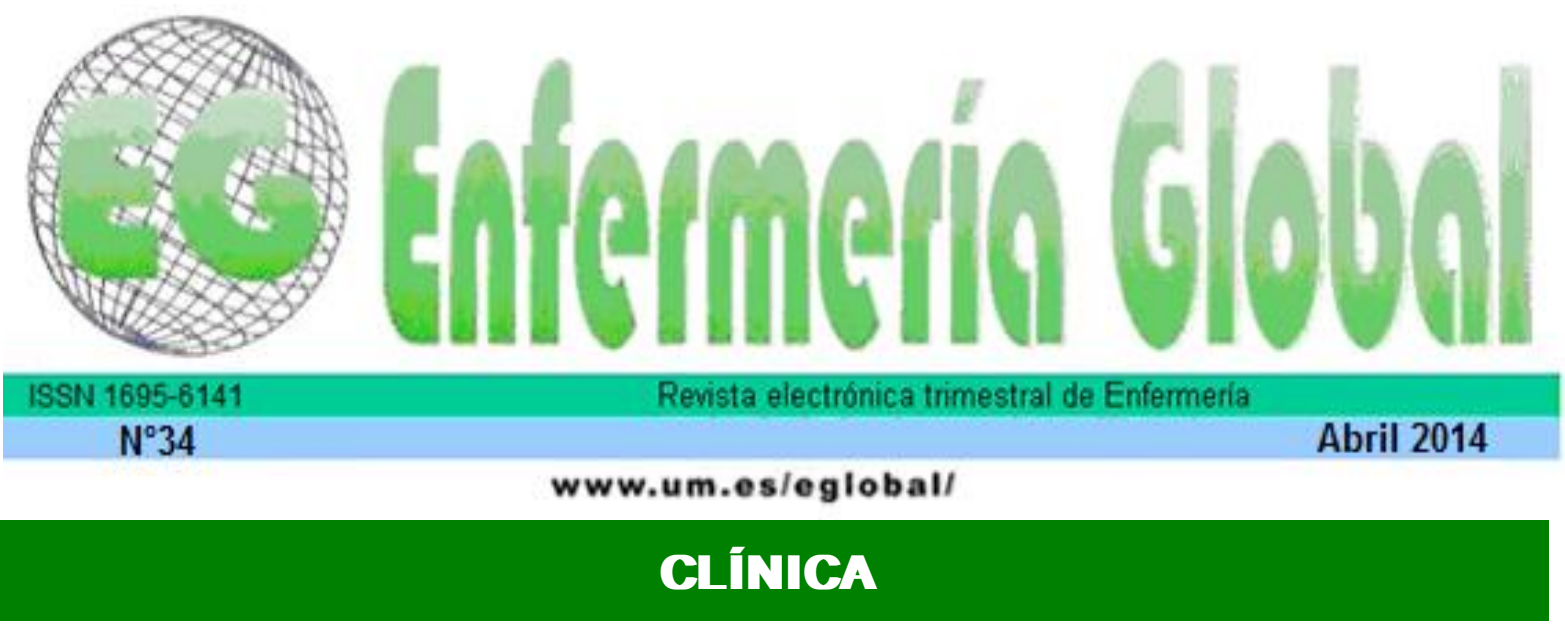

\title{
Evaluación de razones de prevalencia para sedentarismo y factores de riesgo en un grupo de estudiantes universitarios. Chía - Colombia
}

Evaluation of prevalence reasons for inactivity and risk factors in a group of university students. Chía - Colombia

\section{*Moreno Collazos, Jorge Enrique ${ }^{* *}$ Cruz Bermúdez, Harold Fabián ${ }^{* * *}$ Angarita Fonseca, Adriana}

*Fisioterapeuta. Magister en Ciencias de la Actividad Física y Deporte; Especialista en Rehabilitación
Cardiopulmonar. Facultad de Enfermería y Rehabilitación. Universidad de La Sabana. Chía. E-mail:
Jorge.moreno2@unisabana.edu.co **Enfermero. Director de Investigación. Fundación Hematológica
Colombia. Bogotá. ***Fisioterapeuta. Magister en Epidemiologia. Universidad de Santander. Bucaramanga. Colombia.

Palabras clave: Actividad física; Jóvenes universitarios; Sedentarismo (Source: DeCs-Bireme)

Keywords: Physical activity; College youth; Sedentary behaviour.

\section{RESUMEN}

El sedentarismo en la población joven se convierte en un factor que predispone al deterioro de la salud, los factores de riesgo deben controlarse para disminuir el impacto en la población universitaria.

Objetivo: Evaluar razones de prevalencia para sedentarismo y factores de riesgo en un grupo de estudiantes universitarios

Método: Se realizó un estudio trasversal descriptivo en 52 estudiantes de los programas de Fisioterapia, Enfermería y Medicina que asistieron a un programa de promoción de la salud. Se realizó un análisis de regresión de poisson múltiple para el cálculo de razones de prevalencia. EI software usado fue Stata 11.0.

Resultados: Estar entre los 20 y 27 años de edad aumenta 2,7 (IC95\% 1,2-5,7) la prevalencia de sedentarismo comparado con tener entre 17 y 19 años ajustado por riesgo cardiovascular y frecuencia cardiaca. 
Discuusión: En el estudio se establecieron razones de prevalencia entre los factores de riesgo asociados a sedentarismo y factores de riesgo, entre los más importantes fue la edad. Se evidencia un comportamiento importante en relación a la presencia de sedentarismo en los estudiantes.

\section{ABSTRACT}

Physical inactivity in young people becomes a predisposing factor to the deterioration of health; risk factors must be controlled to reduce the impact on the university.

Objective: to evaluate reasons for inactivity prevalence and risk factors in a group of university students.

Methods: a descriptive transversal study conducted on 52 students in the programs of Physiotherapy, Nursing and Medicine who attended a program of health promotion. An analysis of multiple Poisson regression to calculate prevalence ratios. The software used was Stata 11.0.

Results: be between 20 and 27 years old increased 2.7 (95\% Cl 1.2 to 5.7) the prevalence of physical inactivity compared to having between 17 and 19 years adjusted for cardiovascular risk and heart rate.

Discussion: the study established the prevalence ratios between risk factors associated with sedentary lifestyle and risk factors, the most significant was age. We found a significant behavior in relation to the presence of sedentary students

\section{INTRODUCCIÓN}

En general en la población se han presentado cambios importantes en relación a la actividad física, en la actualidad se evidencia un notable incremento en la morbilidad y mortalidad principalmente asociada a enfermedades crónicas como la obesidad, diabetes, hipertensión arterial, enfermedades cardiovasculares entre las más importantes, la mayoría de las cuales se han reportado con asociaciones directas con malos hábitos alimentarios y sedentarismo ${ }^{(1)}$. Por lo anterior, el sedentarismo se enmarca como un factor de riesgo de primer orden para desarrollar enfermedades que afectan a la productividad de la población, afecciones que se pueden prevenir modificando actitudes que ponen en riesgo la salud de la población ${ }^{(2)}$.

En relación al aumento del sedentarismo en la población, la Organización Panamericana de la Salud [OPS] estimó en un estudio en el 2005 que un promedio de 170,000 muertes en América Latina y el Caribe se derivaron de la ausencia de actividad física y comparó en dicho estudio aquellos sujetos con actividad física regular frente a aquellos con hábitos sedentarios encontrando mayor riesgo en aquellos que no practican ningún tipo de actividad física ${ }^{(3)}$.

Cuando hablamos de sedentarismo nos referimos a la falta de actividad física (menos de entre 25 y 30 minutos diarios por 3 días en la semana), autores plantean que un nivel de actividad física moderada es aquella en la cual se realiza más de 30 minutos, 3 días por semana, actividad regular que trae beneficios importantes para la salud del individuo entre los que se han descrito la disminución notablemente de factores de riesgo asociados a problemas cardiovasculares como son la hipercolesterolemia, aumento en la circulación neurológica lo que influye en procesos de aprendizaje y atención; por otra parte el estímulo que genera la realización de actividad física promueve la liberación de 
adrenalina que mejoran el estado de ánimo ${ }^{(4)}$. Diversos estudios colombianos en población universitaria reportan una baja tasa de actividad física en esta población, con valores superiores al $75 \%$ de falta de actividad física ${ }^{(5-6)}$.

El exceso de peso y la obesidad se han relacionado clínicamente de igual manera que el sedentarismo como un precursor en la aparición y padecimiento de enfermedades crónicas, las cuales en la actualidad se han convertido en un problema de interés para las autoridades que regulan la salud pública en el mundo, en el año 2005 la OMS reportó que alrededor de 1600 millones de personas padecen de sobrepeso u obesidad ${ }^{(7)}$. La relación cintura-cadera $[\mathrm{C} / \mathrm{C}]$ es aceptado como un buen indicador de la obesidad central y se ha establecido como un indicador de riesgo cardiovascular en aquella población con los siguientes valores; $>1$ en los hombres y $>0,85$ en las mujeres ${ }^{(8,9)}$.

Existen descritos otros factores definidos como factores socioculturales que afectan directamente la aparición de problemas cardiovasculares en los jóvenes, que a su vez se ven influenciados por un periodo de adaptaciones culturales en búsqueda de un estilo propio de vida y una construcción de su imagen ante los demás; el estrés por ejemplo que generan los procesos de aprendizaje, el cambio en el estilo de vida puede influenciar en niveles de presión arterial la cual se pueden elevar en estados de estrés agudo y asociarse con una mayor predisposición al realizar prácticas de riesgo para la salud como tabaquismo, consumo de alcohol, comer en exceso, entre otros ${ }^{(10,11)}$

Por lo anterior se planteó el siguiente estudio el cual buscó evaluar razones de prevalencia para sedentarismo y factores de riesgo en un grupo de estudiantes universitarios que asistieron a la jornada "Muévete por tu Salud" desarrollada por la facultad de Fisioterapia de la Universidad de la Sabana. Chía - Colombia.

\section{MÉTODO}

Se realizó un estudio de corte transversal inferencial en el periodo de marzo 2012 en la Universidad de la Sabana, Chía - Colombia en la jornada de prevención en salud "Muévete por tu Salud" realizada por el programa de Fisioterapia. La recolección de la muestra fue no probabilística teniendo en cuenta 52 estudiantes de los programas de Fisioterapia, Enfermería y Medicina que asistieron voluntariamente a la jornada. Dentro de los criterios de inclusión se determinó ser estudiante activo de la universidad. Los criterios de exclusión fueron sujetos que no quisieran participar en el estudio.

Para la obtención de las variables fisiológicas se realizó la medición de la Frecuencia cardiaca, frecuencia respiratoria, Tensión arterial sistólica y diastólica. Con los valores de la variable Tensión arterial se generó la clasificación de hipertensión según la definición de la Sociedad colombiana de cardiología, Guías colombianas para el diagnóstico y tratamiento de la hipertensión arterial ${ }^{(12)}$. En la consecución de medidas antropométricas se evaluó; talla, peso, perímetro de cintura y perímetro de cadera, se calculó la variable Índice de masa corporal [IMC] según la ecuación $\mathrm{IMC}=$ Peso [Kilogramos] / Talla ${ }^{2}$ [metros] y la relación cinturacadera [ICC], con la formula ICC= cintura [centímetros] Cadera [centímetros]. Las otras variables fueron tomadas por medio de un interrogatorio, género, edad, carrera, sedentarismo definida como Falta de actividad física menos de 30 minutos y de 3 veces por semana, consumo de cigarrillo, para esta última no se indagó por el número y la frecuencia de cigarrillos consumidos, la variable exceso 
de peso se definió en relación a la interpretación del IMC según lo establecido por la OMS.

Los instrumentos utilizados para le medición de las variables fisiológicas fueron: tensiómetro de brazo profesional Referencia 79 Prestigie Medical, tallimetro y cinta de medida en centímetros. La medición de las variables fisiológicas se tomó por un profesional debidamente capacitado en la competencia.

El desarrollo de la investigación contó con el aval de la institución y se enmarca en la categoría de investigación con riesgo mínimo según la resolución No. 8430 de 1993 del Ministerio de Salud de Colombia, que establece las normas científicas, técnicas y administrativas para la investigación en salud. Los sujetos participantes del estudio aceptaron la participación en el mismo y firmaron consentimiento informado.

\section{Análisis estadístico}

Inicialmente se realizó el análisis descriptivo, se calcularon promedios y desviaciones estándar para las variables cuantitativas y frecuencias absolutas y relativas e intervalos de confianza del 95\% (IC95\%) para las variables cualitativas. En el análisis bivariado se aplicó un prueba t de student e intervalos de confianza del 95\% (IC95\%), se realizó un análisis entre sujetos con sedentarismo frente al global de la población para las variables fisiológicas y medidas antropométricas. En el análisis multivariado se realizó un análisis de regresión de poisson simple para el cálculo de razones de prevalencia en donde se incluirán las variables del estudio, se analizaran las variables del modelo final. En todo el análisis se utilizó un nivel alpha de 0,05. El software usado fue Stata 11.0.

\section{RESULTADOS}

La población de estudio estuvo conformada por 52 estudiantes, la mayoría entre 17 y 19 años (57,7\%), de género femenino (80,8\%) y de la carrera de Fisioterapia $(69,2 \%)$ Tabla 1.

Tabla 1. Descripción de la población de estudio.

\begin{tabular}{llcc}
\hline \multicolumn{2}{c}{ Variable } & $\mathrm{n}$ & $\%$ \\
\hline Edad & $17-19$ & 30 & 57,7 \\
(agrupado) & $20-27$ & 22 & 42,3 \\
Genero & Masculino & 10 & 19,2 \\
& Femenino & 42 & 80,8 \\
Carrera & Enfermería & 15 & 28,9 \\
& Fisioterapia & 36 & 69,2 \\
& Medicina & 1 & 1,9 \\
\hline
\end{tabular}

Se evaluaron factores de riesgo a sedentarismo en la muestra, se encontró que los estudiantes de mayor edad (20 a 27 años) presentan una prevalencia de sedentarismo de 2,5 (IC95\% 1,2-5,2) veces comparado con los estudiantes de menor edad (17-19 años). Adicionalmente quienes tienen riesgo para la salud según el perímetro de cintura presentaron una prevalencia de 2,12 (IC95\% 1,074,19 ) veces comparado con los estudiantes que no tienen riesgo Tabla 2. 
Tabla 2. Factores de riesgo asociados a sedentarismo en una población de estudiantes universitarios.

\begin{tabular}{|c|c|c|c|c|c|}
\hline \multirow{2}{*}{ Variable } & \multicolumn{2}{|c|}{ Sedentarismo } & \multirow{2}{*}{$\mathrm{RP}^{*}$} & \multirow{2}{*}{ IC95\% } & \multirow{2}{*}{$\begin{array}{l}\text { Valor } \\
\text { p }\end{array}$} \\
\hline & $\mathrm{Si}$ & No & & & \\
\hline \multicolumn{6}{|l|}{$\begin{array}{l}\text { Edad (Agrupada } \\
\text { por años) }\end{array}$} \\
\hline $17-19$ & $7(23,3)$ & $23(76,7)$ & 1 & & \\
\hline $20-27$ & $13(59,1)$ & $9(40,9)$ & 2,5 & $(1,2-5,2)$ & 0,009 \\
\hline \multicolumn{6}{|l|}{ Género } \\
\hline Femenino & $16(38,1)$ & $26(61,9)$ & 1 & $(0,25-1,92)$ & \\
\hline Masculino & $4(40,0)$ & $6(60,0)$ & 1,05 & $(0,45-2,46)$ & 0,911 \\
\hline \multicolumn{6}{|l|}{ Carrera } \\
\hline Fisioterapia & $14(38,9)$ & $22(61,1)$ & 1 & & \\
\hline Enfermería/Medicina & $6(37,5)$ & $10(62,5)$ & 0,96 & $(0,45-2,04)$ & 0,924 \\
\hline \multicolumn{6}{|l|}{ Tabaquismo } \\
\hline No & $15(34,9)$ & $28(65,1)$ & 1 & & \\
\hline $\mathrm{Si}$ & $5(55,6)$ & $4(44,4)$ & 1,59 & $0,78-3,25$ & 0,246 \\
\hline \multicolumn{6}{|l|}{ Alcohol } \\
\hline No & $20(39,2)$ & $31(60,8)$ & 1 & & \\
\hline $\mathrm{Si}$ & $0(0,0)$ & $1(100,0)$ & - & - & - \\
\hline \multicolumn{6}{|l|}{ Exceso de peso } \\
\hline No & $17(39,6)$ & $27(61,4)$ & 1 & & \\
\hline $\mathrm{Si}$ & $3(37,5)$ & $5(62,5)$ & 0,97 & $(0,37-2,56)$ & 0,952 \\
\hline \multicolumn{6}{|l|}{$\begin{array}{l}\text { Riesgo } \\
\text { Cardiovascular } \\
\text { según relación } \\
\text { cintura Cadera }\end{array}$} \\
\hline No & $13(38,2)$ & $21(61,8)$ & & & \\
\hline $\mathrm{Si}$ & $7(38,9)$ & $11(61,1)$ & 1,01 & $(0,50-2,09)$ & 0,963 \\
\hline \multicolumn{6}{|l|}{$\begin{array}{l}\text { Riesgo } \\
\text { Cardiovascular } \\
\text { según perímetro } \\
\text { de cintura }\end{array}$} \\
\hline No & $17(35,4)$ & $31(64,6)$ & 1 & & \\
\hline $\mathrm{Si}$ & $3(75,0)$ & $1(25,0)$ & 2,12 & $(1,07-4,19)$ & 0,118 \\
\hline \multicolumn{6}{|l|}{$\begin{array}{l}\text { Hipertensión } \\
\text { arterial }\end{array}$} \\
\hline No & $19(38,8)$ & $30(61,2)$ & & & \\
\hline $\mathrm{Si}$ & $1(33,3)$ & $2(66,7)$ & 0,85 & $(0,17-4,43)$ & 0,35 \\
\hline
\end{tabular}

En la Tabla 3, se observa que los estudiantes sedentarios tienen una frecuencia cardíaca mayor (81,8 $\pm 11,3$ ppm) comparada con los estudiantes no sedentarios 
$(74,2 \pm 13,5 p p m)(p=0,040)$. En las demás variables no se encontraron diferencias estadísticamente significativas entre sedentarios y no sedentarios.

Tabla 3. Variables clínicas asociadas a sedentarismo (Cuantitativas) en una población de estudiantes universitarios.

\begin{tabular}{|c|c|c|c|c|c|c|c|}
\hline \multirow{3}{*}{ Variable } & \multicolumn{4}{|c|}{ Sedentarismo } & \multicolumn{3}{|c|}{ Global } \\
\hline & \multicolumn{2}{|c|}{ SI } & \multicolumn{2}{|c|}{ NO } & \multirow[b]{2}{*}{ Promedio } & \multirow[b]{2}{*}{$\begin{array}{c}\text { Desviación } \\
\text { Estándar }\end{array}$} & \multirow[b]{2}{*}{$\begin{array}{c}\text { Valor } \\
p^{*}\end{array}$} \\
\hline & Promedio & $\begin{array}{l}\text { Desviación } \\
\text { Estándar }\end{array}$ & Promedio & $\begin{array}{l}\text { Desviación } \\
\text { Estándar }\end{array}$ & & & \\
\hline $\begin{array}{l}\text { Frecuencia } \\
\text { cardiaca }\end{array}$ & 81,8 & 11,3 & 74,1 & 13,5 & 77,1 & 13,2 & 0,04 \\
\hline $\begin{array}{l}\text { Frecuencia } \\
\text { respiratoria }\end{array}$ & 18,6 & 1,8 & 18,3 & 1,9 & 18,4 & 1,83 & 0,697 \\
\hline $\begin{array}{l}\text { Tensión } \\
\text { arterial } \\
\text { sistólica }\end{array}$ & 112,9 & 8,1 & 118,1 & 10,3 & 116,1 & 9,74 & 0,058 \\
\hline $\begin{array}{l}\text { Tensión } \\
\text { arterial } \\
\text { diastólica }\end{array}$ & 78 & 8,3 & 77,3 & 7,4 & 77,6 & 7,7 & 0,768 \\
\hline Talla & 1,64 & 0,1 & 1,65 & 0,1 & 1,65 & 0,08 & 0,751 \\
\hline Peso & 59,5 & 10,4 & 59,7 & 9,8 & 59,6 & 9,9 & 0,946 \\
\hline $\begin{array}{l}\text { Índice de } \\
\text { Masa } \\
\text { Corporal }\end{array}$ & 23 & 3 & 21,9 & 3 & 21,9 & 3 & 0,9 \\
\hline $\begin{array}{l}\text { Perímetro } \\
\text { de cintura }\end{array}$ & 75,4 & 10,5 & 75,5 & 8 & 75,4 & 8,9 & 0,984 \\
\hline $\begin{array}{l}\text { Perímetro } \\
\text { de cadera }\end{array}$ & 95,3 & 7,5 & 96,3 & 6,1 & 95,9 & 6,6 & 0,584 \\
\hline $\begin{array}{l}\text { Relación } \\
\text { cintura } \\
\text { cadera }\end{array}$ & 0,79 & 0,1 & 0,78 & 0,1 & 0,79 & 0,06 & 0,708 \\
\hline
\end{tabular}

${ }^{\text {*Prueba } t \text { de student }}$

El análisis de regresión de poisson múltiple, mostró que estar entre los 20 y 27 años de edad aumenta 2,7 (IC95\% 1,2-5,7) la prevalencia de sedentarismo comparado con tener entre 17 y 19 años ajustado por riesgo cardiovascular y frecuencia cardiaca Tabla 4.

Tabla 4. Factores de riesgo asociados a sedentarismo.

Modelo final.

\begin{tabular}{lccc}
\hline \multicolumn{1}{c}{ Variable } & $\mathrm{RP}^{*}$ & $\mathrm{IC} 95 \%$ & Valor $\mathrm{p}$ \\
\hline Edad (agrupado) & & & \\
$17-19$ & 1 & & \\
$20-27$ & 2,7 & $\left(\begin{array}{c}(1,2- \\
5,7)\end{array}\right.$ & 0,012 \\
\hline
\end{tabular}

Riesgo

Cardiovascular

según perímetro

de cintura

No 


\begin{tabular}{lccc}
$\mathrm{Si}$ & 2,5 & $\begin{array}{c}(0,90- \\
6,8)\end{array}$ & 0,078 \\
\hline $\begin{array}{l}\text { Frecuencia } \\
\text { cardiaca }\end{array}$ & 1,02 & $\begin{array}{c}(0,99- \\
1,05)\end{array}$ & 0,151 \\
\hline
\end{tabular}

${ }^{\star}$ Regresión de poisson

\section{DISCUSIÓN}

En el estudio se establecieron razones de prevalencia entre los factores de riesgo asociados a sedentarismo y factores de riesgo en un grupo de estudiantes universitarios de programas de ciencias de la salud que asistieron a un programa de tamizaje para riesgo cardiovascular y sedentarismo organizado por la facultad de enfermería y rehabilitación.

En relación a la distribución por género, se evidenció un mayor porcentaje en el género femenino para la clasificación de sedentarismo, por otra parte en general para la distribución de las variables del estudio se encontró un comportamiento similar a lo reportado en otros estudios realizados en población universitaria ${ }^{(13,14)}$.

En el cálculo de prevalencia de sedentarismo se encontró que el grupo de estudio con edades mayores a 20 años tiene mayor influencia frente a los menores de esta edad, tal vez este comportamiento se explica por la innovación de la tecnología, la falta de tiempo para realizar actividad física, obligaciones académicas, el incremento en el uso automóviles y motos para el desplazamiento como los factores más determinantes y relacionados con la edad según lo descrito por otros estudios ${ }^{(15-17)}$. Por otro lado se evidencia un mayor riesgo en la población de estudio en la variable "medición del perímetro de cintura", ya que esta clínicamente se encuentra asociada al depósito de grasa en el abdomen y a características de obesidad que se han descrito en la literatura como un importante factor de riesgo para la salud ${ }^{(8,9)}$. Se detectó que los estudiantes sedentarios tienen una frecuencia cardíaca mayor comparada con los estudiantes no sedentarios, situación que se puede relacionar como un signo de afectación del sistema cardiovascular, aunque simplemente se analiza como una variable fisiológica.

El consumo de tabaco y alcohol no se estimó como prevalencia de riesgo, situación explicada por el tamaño de la muestra y por la no identificación de la frecuencia de consumo, en otros estudios se ha descrito como un factor de riesgo y se debe esclarecer que este en realidad se comporta como un hábito importante que desencadena procesos de enfermedad en la población ${ }^{(18-19)}$.

\section{CONCLUSIONES}

La prevalencia de sedentarismo comparado con edad se comporta como una variable de interés, los programas de promoción de la actividad física se centran en mejorar las percepciones de la población frente al ejercicio regular como un hábito y estilo de vida saludable, influyendo directamente en los factores de riesgo cardiovasculares. Es importante la incorporación de programas de promoción y prevención encaminados a disminuir los factores de riesgo asociados al sedentarismo, programas en donde la participación de enfermería y fisioterapia juegan un papel importante. Por otra parte se recomienda para futuros trabajos evaluar la exposición a factores de riesgo en relación al tiempo, usando 
mediciones en varios periodos de tiempo, de igual manera buscar determinar no solo el factor de riesgo sino la causalidad del mismo.

\section{Agradecimientos}

Al grupo de estudiantes de la Facultad de Fisioterapia de la Universidad de la Sabana, quienes participaron en la organización del evento "Muévete por tu salud", docentes y administrativos que permitieron la logística del mismo.

\section{REFERENCIAS BIBLIOGRÁFICAS}

1. Jadue L, Vega J, Escobar M. Factores de riesgo para enfermedades no transmisibles: metodología y resultados globales de la encuesta de base del programa Carmen. Rev Méd Chil 1999; 127: 1004-1013.

2. Mahecha Matsudo Sandra, Rodrigues Matsudo Victor, et.al. Physical Activity Promotion: Experiences and Evaluation of the Agita Sao Paulo Program Using the Ecological Mobile Model. Journal of Physical Activity and Health, 2004, 1, 81-87.

3. Organización Panamericana de la Salud [sede web]. Washington, US. 2007. [fecha de acceso noviembre 27 de de 2008]. Salud en las Américas. Disponible en http://www.per.opsoms.org/sea-07/SEA-2007-3.pdf

4. Hillman $\mathrm{CH}$, Erickson $\mathrm{KI}$, Kramer AF. Be smart, exercise your heart: Exercise effects on brain and cognition. Nat Rev Neurol. 2008; 9:58-65.

5. Gómez LF, Mateus JC, Cabrera G. Leisure-time physical activity among women in a neighbourhood in Bogotá, Colombia: Prevalence and sociodemographic correlates. Cad Saude Publica. 2004; 20: 1103-9.

6. Lema LF, Salazar IC, Varela MT, Díaz JA, Rubio A, Botero A. Comportamiento y salud de los jóvenes universitarios: Satisfacción con el estilo de vida. Pens Psicol. 2009; 5: 71-88.

7. Aranceta- Bartrina, J. et al. Prevalencia de obesidad en España. Unidad de Nutrición Comunitaria, Med. Clin. 2005 (disponible en: http://www.seedo.es/portals/seedo/consenso/

Prevalencia_obesidad_Espa\%C3\%

8. Bray G, Bouchard $\bar{C}$, James WPT. Definitions and proposed current classifications of obesity. En: Bray G, Bouchard C, James WPT, editores. Handbook of obesity. Nueva York: Marcek Dekker, 1998; 31-40.

9. NIH. National Institutes of Health. National Heart, Lung and Blood Institute.Clinical guidelines on the identification, evaluation, and treatment of overweight and obesity in adults. The evidence report. Bethesda, junio 1999.

10. Grisales H, Caicedo B, Serna C, Uribe D. Causas de mortalidad en jóvenes y su contribución al cambio en la esperanza de vida: Cali, 1989-1999. Colomb Med 2005; 36(2):85-93.

11. Campo J, Reyes J, Ortiz C. Blood pressure levels and psychosocial stress in students of health sciences. University of Valle, Cali, Colombia, 20032004. Colomb Med 2006; 37(2 suppl.1):21-25.

12. Nubia Roa B. Definición - Diagnóstico de la hipertensión arterial. Revista colombiana de Cardiología 2007; 13 (1) 195-196

13. Pavón A, Moreno JA. Actitud de los universitarios ante la práctica físicodeportiva: Diferencias por géneros. Rev Psicol Deport. 2008; 17: 7-23.

14. Hernán M, Ramos M, Fernández A. Salud y juventud. Madrid: Consejo de la Juventud de España, Escuela Andaluza de Salud Pública; 2002. 
15. Moya-Sinfontes M, García P, Lucena N, Casañas R, Brito P, Rodríguez A, et al. Hipocinetismo: un problema de salud entre jóvenes ucevistas? RFM 2006; 29(1).

16. Romo R, Cerda M. Estilo de vida en los estudiantes universitarios de la Facultad de Medicina de la Universidad de Chile. Rev Salud Pública Méx 2002; 7(11):14-25.

17.Gutiérrez YJ, Vargas NS [Internet]. Disponible en: http://www.fepafem.org.ve/investigacionesinvestigaciones2003/art1a.htm. Consultado Enero del 2012.

18. Celermajer D, Adams M, Clarkson P, Robinson J, McCredie R, Donald A, et al. Passive smoking and impaired endothelium-dependent arterial dilatation in healthy young adults. NEJM 1996; 334:150-154.

19. Johnson C, Myers L, Webber L, Hunter S, Srinivasan S, Berenson G. Alcohol consumption among adolescents and young adults: the Bogalusa Heart Study 1981 to 1991. Am J Public Health 1995; 85:979-982. 